\title{
Thrombosis and Hemorrhage in Diabetic Retinopathy: A Perspective from an Inflammatory Standpoint
}

\author{
Nivetha Murugesan, $\mathrm{PhD}^{1,2}$ Tuna Üstunkaya, BSc ${ }^{3}$ \\ ${ }^{1}$ Section on Vascular Cell Biology, Research Division, Joslin Diabetes \\ Center, Boston, Massachusetts \\ 2 Department of Medicine, Harvard Medical School, Boston, Massachusetts \\ ${ }^{3}$ Hacettepe University Medical School, Ankara, Turkey
}

Edward P. Feener, PhD ${ }^{1,2}$

\begin{abstract}
Keywords

- thrombosis

- hemorrhage

- diabetic retinopathy

- inflammation
\end{abstract}

Diabetic retinopathy is a leading cause of vision loss in working-age adults. ${ }^{1}$ Nearly all people with 20 or more years of diabetes develop at least early stages of nonproliferative diabetic retinopathy (NPDR), which are characterized by microvascular abnormalities, including microaneurysms, retinal vascular hyperpermeability, exudates, and intraretinal "dot" hemorrhages. Worsening of these microvascular lesions associated with severe NPDR can lead to retinal ischemia and neovascularization, termed proliferative diabetic retinopathy (PDR). In addition, diabetic retinopathy can also cause diabetic macular edema (DME), which is characterized by an accumulation of fluid in the macular region, resulting in impaired central vision. Although early stages of diabetic retinopathy have been primarily attributed to the effects of
Address for correspondence Edward P. Feener, PhD, Section on Vascular Cell Biology, Research Division, Joslin Diabetes Center, One Joslin Place, Boston, MA 02215

(e-mail: Edward.Feener@joslin.harvard.edu). hyperglycemia, hypertension, and dyslipidemia, ${ }^{2}$ secondary intraocular responses play a critical role in disease progression to PDR and DME. Advanced stages of diabetic retinopathy are frequently associated with regions of reduced capillary perfusion, evident by fluorescein angiography, which result in hypoxia-induced expression of vascular endothelial growth factor (VEGF). Increased intraocular concentrations of VEGF in ischemic retinopathies play important roles in both retinal neovascularization and macular edema. ${ }^{3}$ Therefore, a key stage in the pathogenesis of diabetic retinopathy to its sight-threatening stages involves the progression from microvascular abnormalities in NPDR to a critical threshold of reduced retinal perfusion leading to retinal ischemia. The appearance of retinal ischemia and hemorrhages has published online August 25, 2015
Issue Theme Inflammation, Endothelial Dysfunction, and Thromboembolism; Guest Editors: Bashir A. Lwaleed, PhD, FRCPath, Rashid S. Kazmi, MRCP, FRCPath, and Alan J. Cooper, PhD.
Copyright $\odot 2015$ by Thieme Medical Publishers, Inc., 333 Seventh Avenue, New York, NY 10001, USA. Tel: +1(212) 584-4662.
DOI http://dx.doi.org/ 10.1055/s-0035-1556731. ISSN 0094-6176. 
implicated roles for coagulation and fibrinolytic cascades in the pathogenesis of diabetic retinopathy. However, while the effects of diabetes and hyperglycemia on coagulation and fibrinolytic systems, and their respective roles in thrombosis, have been well documented, ${ }^{4,5}$ relatively little is known about the impact of these systems on diabetic retinopathy. In addition, components of these systems also exert potent effects on inflammation, which may also contribute to the progression of diabetic retinopathy to DME independently of retinal ischemia. ${ }^{6}$ This article examines the potential interactions between intraocular thrombotic and inflammatory systems in the pathogenesis of diabetic retinopathy.

\section{Inflammation, Thrombosis, and Retinal Ischemia}

At the cellular level, chronic diabetes induces retinal pericyte loss and capillary endothelial cell apoptosis, which impairs blood retinal barrier function and blood flow. ${ }^{7}$ These changes have been associated with the formation of acellular capillaries, which have been observed in trypsin digests of retinal microvascular tissue obtained from diabetic experimental animal models. ${ }^{8}$ Several mechanisms, including upregulation of the angiopoietin-2/Tie2 ligand/receptor system, have been implicated in contributing to pericyte loss and increased acellular capillary segments in retina from diabetic rodents. ${ }^{9}$ However, less is known about the processes that may contribute to degenerate capillaries in diabetic human retina and their significance in the transition from NPDR to ischemia retinopathy.

Diabetic retinopathy in animal models is associated with inflammation, including, for example, increased retinal expression of intercellular adhesion molecule-1 (ICAM-1) and leukocyte adhesion in capillaries and venules. ${ }^{10}$ Leukocyte $\alpha 4$ integrin/vascular cell adhesion molecule (VCAM-1) signaling is also implicated in leukocyte adhesion and vascular leakage in diabetic retinopathy. Blockade of $\alpha_{4}$ integrin reduces retinal levels of VEGF, TNF $\alpha$, and NF-KB activity, as well as retinal vascular hyperpermeability and leukocyte adhesion in diabetic rats. ${ }^{11}$ Although hyperglycemia-induced leukostasis has been implicated in retinal capillary apoptosis and occlusion in animal models of diabetic retinopathy, ${ }^{10}$ the significance of this mechanism to ischemic diabetic retinopathy, PDR, and DME is not yet available. Insulin resistance and hypertension also increase leukocyte binding to the retinal endothelium, ${ }^{12,13}$ without evidence of ischemic retinal disease, suggesting that chronic effects on leukostasis are not sufficient to cause significant retinal ischemia. A recent report has shown that retinal ICAM-1 expression and leukostasis are not altered in moderately controlled hyperglycemia (HbA1c 6-10\%) and leukostasis in retinal arteries is decreased in a rodent model of type 2 diabetes with severe hyperglycemia (HbA1c >10\%). ${ }^{14}$ Moreover, diabetes in humans can occur for decades before or without onset of ischemic retinopathy. Thus, it is unclear how the chronic effects of diabetes on leukostasis explain the onset of retinal ischemia in the progression from NPDR to PDR for a subset of affected patients. As mentioned earlier, the transition of diabetic retinopathy to PDR and DME is also associated with increased intraocular concentrations of VEGF. Intravitreal injection of VEGF induces retinal vascular leakage, hemorrhage, and leukostasis. ${ }^{15,16}$ Repeated intravitreal injections of VEGF in primates cause retinal capillary nonperfusion and neovascularization. ${ }^{17} \mathrm{~A}$ recent study has reported that intravitreal administration of ranibizumab, an anti-VEGF monoclonal antibody, reduced, but did not eliminate, capillary nonperfusion in DME patients. ${ }^{18}$ These findings suggest that VEGF, as well as potentially other cytokines and metabolic factors, induces thromboinflammatory processes in the retina that contribute to capillary nonperfusion and ischemia.

Diabetes is a procoagulate state and retinal microthromboses occur in diabetic retinopathy. Increased prevalence of platelet-fibrin microthrombi positive for factor XIII immunostaining has been observed in the retinal microvasculature both in donor human eyes from diabetic subjects and in experimental rodent models of diabetes, compared with their respective controls. ${ }^{19,20}$ Although these microthrombi are associated with capillary and venule occlusion, their significance to retinal ischemia, PDR, and DME is not yet available. It is well established that diabetes induces endothelial dysfunction and injury, which can lead to increased expression of proinflammatory adhesion molecules, apoptosis, and increased leukocyte and platelet adhesion in the retinal microvasculature. ${ }^{10}$ Immunohistochemical analyses show that microthrombi are colocalized with apoptotic retinal endothelial cells, ${ }^{19,20}$ suggesting that microthromboses are concomitant with or secondary to capillary damage. Blood platelets are more likely to adhere to the diabetic endothelium than healthy vessels, ${ }^{21}$ which may contribute to the occlusion of injured retinal capillaries. In a cross-sectional study of 227 patients with type II diabetes mellitus (119 with vs 108 without diabetic retinopathy) and 169 nondiabetic subjects, it was demonstrated that polymorphism in platelet $\alpha_{2} \beta_{1}$, a receptor for collagen important for platelet adhesion and thrombus formation, is an independent risk factor for retinopathy. ${ }^{22}$ Taken together, platelet adhesion to the injured diabetic endothelium may contribute to both ischemia and inflammation in diabetic retinopathy.

\section{Retinal Hemorrhage and Inflammation}

Diabetic retinopathy is also characterized by the presence of small "dot" intraretinal hemorrhages. The number and severity of these hemorrhages are frequently increased during progression from mild to moderate to severe stages of NPDR. The occurrence of these hemorrhages has been attributed, in part, to vascular hyperpermeability and microaneurysms. Although diabetes increases retinal vascular permeability via the disruption of tight junction integrity in the retinal endothelium, the loss of tight junction function alone may not be sufficient to fully explain these dot hemorrhages. Intraretinal hemorrhages require disruption of adherens junctions and breakdown of the basement membrane for erythrocytes to escape from the vasculature. In addition to these small intraretinal hemorrhages, larger flame-type hemorrhages can occur in diabetic retinopathy, especially in the presence 
of hypertension, which is an important risk factor for retinopathy progression. Moreover, nascent vessels that are generated during PDR are fragile, usually grow anteriorly into the vitreous, and are vulnerable to rupture and hemorrhage. Indeed, PDR can cause vitreous hemorrhage, leading to vitreoretinal disorders and tractional retinal detachment. Although intraocular hemorrhages occur at multiple levels in diabetic retinopathy and are commonly associated with advance stages of the disease, the effects of hemorrhage on retinopathy progression are not fully understood.

Proteomic analyses of vitreous fluid obtained from patients with advanced diabetic retinopathy have revealed that intraocular hemorrhage and plasma extravasation markedly alter the protein composition of the vitreous humor. $^{23,24}$ These studies have identified components of both coagulation and fibrinolytic cascades in the vitreous in diabetic retinopathy including prothrombin, factor $\mathrm{V}$, factor XII (FXII), fibrinogen, antithrombin III, $\alpha_{1}$-antitrypsin, and heparin cofactor 2 (-Fig. 1). In addition to their roles in thrombosis and hemostasis, these coagulation and fibrinolytic factors increase inflammatory responses that have been implicated in diabetic retinopathy. We identified increased levels of the contact activation system components, including plasma kallikrein, FXII, and high-molecular-weight kininogen
(HK) in the vitreous from patients with advanced diabetic retinopathy. ${ }^{23}$ We have also reported that intravitreal injection of plasma kallikrein exerts potent effects on retinal vascular hyperpermeability, leukostasis, and edema. $23,25,26$ HK and FXII are plasma kallikrein's primary substrates. Cleavage of HK by plasma kallikrein results in the production of bradykinin and thereby activation of $\mathrm{B} 1$ and $\mathrm{B} 2$ receptors, which are $G$ protein-coupled receptors that contribute to retinal vascular inflammation and dysfunction in diabetes. ${ }^{6}$ In addition, several additional substrates for this serine protease have been identified in the secretome from astrocytes and pericytes, including extracellular matrix (ECM) proteins. $^{26,27}$ Liu et al have demonstrated that plasma kallikrein binds ECM, which interferes with collagen-induced platelet activation and impairs cerebral hemostasis in diabetes. ${ }^{28}$ Abdallah et al $^{29}$ have shown that plasma kallikrein also activates protease activated receptor (PAR) 1 and 2 on vascular smooth muscle cells, resulting in increased TNF $\alpha$ release. Intravitreal injections of plasma kallikrein in rats induce retinal hemorrhages, which appears similar to intraretinal dot hemorrhages observed in NPDR. ${ }^{26}$ Plasma kallikrein also causes proteolysis of ECM components including collagen IV, a major constituent of the basement membrane. ${ }^{26,27}$ The collagenase-like activity of plasma kallikrein may contribute

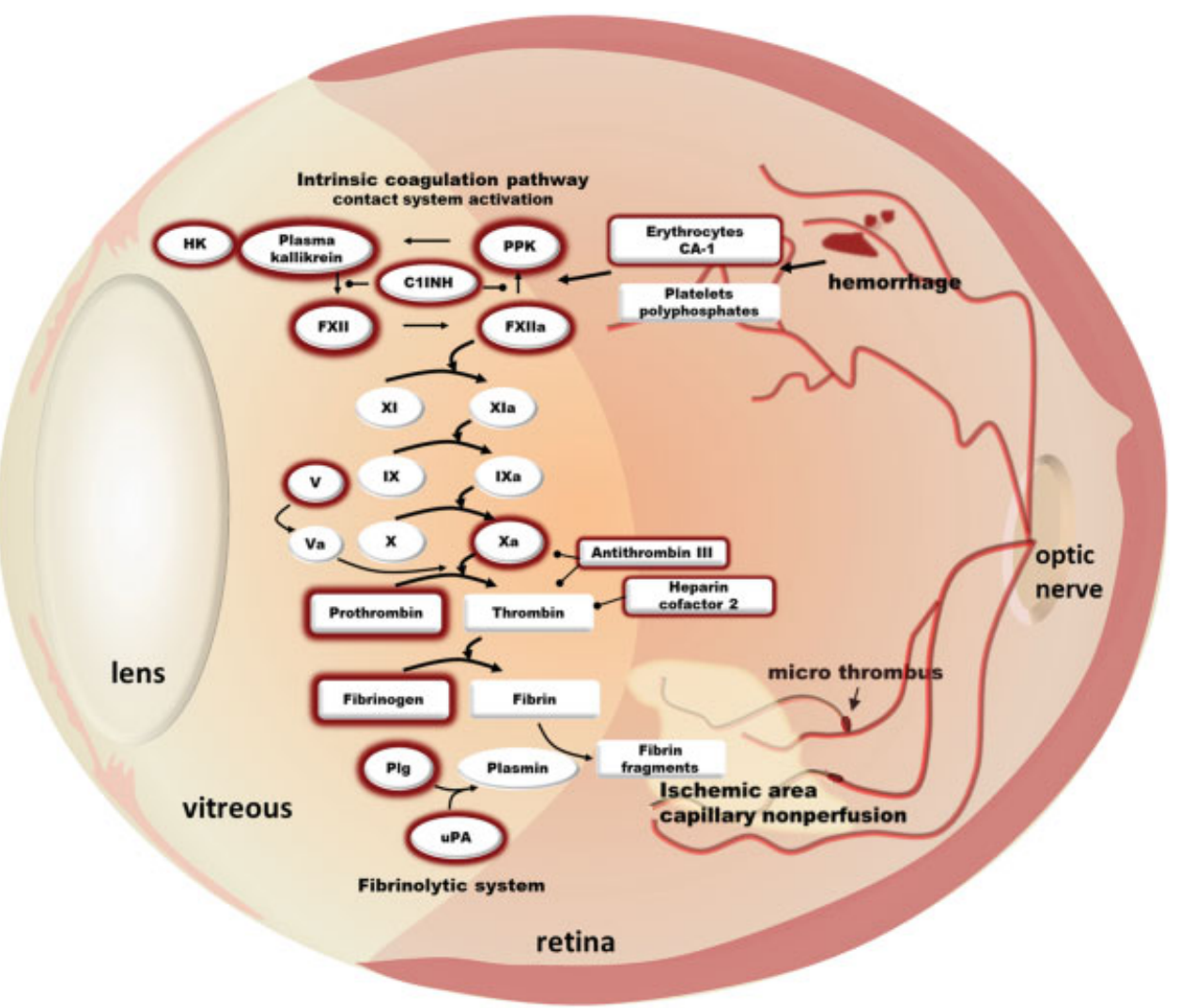

Fig. 1 Intraocular coagulation and fibrinolytic cascades in diabetic retinopathy. Advanced diabetic retinopathy is characterized by the presence of intraretinal hemorrhage and capillary nonperfusion, mediated in part by leukostasis and microthrombi. Intraocular hemorrhage and plasma extravasation markedly alter vitreous protein composition in patients with advanced diabetic retinopathy. Increased levels of coagulation and fibrinolytic factors has been identified in the vitreous of patients with advanced diabetic retinopathy including plasma kallikrein (PK), factor XII (FXII), prothrombin, and urokinase plasminogen activator (UPA) (outlined in dark red). Other components detected also include high-molecularweight kininogen (HK), plasma prekallikrein (PPK), C1 inhibitor (C1INH), carbonic anhydrase 1 (CA-1), and plasminogen (Plg). 
to degradation of ECM and weakening of the basement membrane, thereby increasing susceptibility to vascular rupture and hemorrhage. Thus, the inflammatory effects of the contact activation system appear to be mediated by both bradykinin-dependent and bradykinin-independent mechanisms. The vitreous in PDR also contains remnants of lysed erythrocytes, including carbonic anhydrase 1 and platelet and endothelial microparticles, ${ }^{23,30}$ which have been implicated in activating the contact system and thrombin. ${ }^{31}$ Gao et al have shown that intraocular hemorrhage results in the release of carbonic anhydrase 1 from erythrocytes, leading to increased plasma kallikrein catalytic activity. ${ }^{23}$ This response was inhibited by either $\mathrm{C} 1$ inhibitor, the primary endogenous serpin serine protease inhibitor of the kallikrein-kinin system, or bradykinin BK1 and BK2 receptor antagonists. These findings indicate that the presence of lysed erythrocyte components within local ocular hemorrhage can trigger subsequent contact system components mediating retinal vascular damage in diabetic retinopathy. As mentioned above, diabetic retinopathy is also associated with platelet microparticles and adherent platelets, which may release polyphosphates and trigger FXII-mediated contact system activation. $^{32}$ These pathways could contribute, in part, to the effects of microthrombosis and hemorrhage on retinal inflammation.

In addition to the contact system, several other coagulation factors have been implicated in contributing to retinal inflammation in diabetic retinopathy. Proteomic data revealed that prothrombin is increased approximately 5 -fold and fibrinogen ( $\alpha, \beta$ and gamma chain) levels were elevated more than 11 -fold in the vitreous obtained from patients with PDR compared with nondiabetic subjects. ${ }^{24} \mathrm{~A}$ recent report has implicated increased thrombin activity within the human vitreous of proliferative vitreoretinopathy patients on retinal inflammation. ${ }^{33}$ Specifically, retinal pigmented epithelial cells exposed to vitreous samples containing high thrombin activity resulted in increased production of
CCL2, CXCL8, and IL-6. Further studies demonstrated that thrombin and factor Xa stimulates IL-6, IL-8, MCP-3, and GMCSF production by retinal pigmented epithelial cells via a PAR1 and NF-KB pathway. ${ }^{34}$ Moreover, thrombin-mediated activation of PAR-1 and NF-KB has also been shown to increase VCAM-1 and ICAM-1 expression-two key adhesion molecules implicated in leukocyte adhesion in diabetic retinopathy. ${ }^{10,35}$

Urokinase plasminogen activator (uPA), a component of the fibrinolytic system, is increased in epiretinal membranes obtain from patients with $\mathrm{PDR}^{36}$ and retinal $\mathrm{UPA}$ receptor (UPAR) expression is increased in diabetic rats. ${ }^{37}$ Upon activation, uPA converts plasminogen to plasmin, which mediates fibrinolysis and can activate matrix metalloproteinases. In addition, activation of the UPA/UPAR pathway also contributes to increased retinal vascular permeability in diabetic rats through proteolytic disruption of VE-cadherin. ${ }^{38}$ El-Remessy et $\mathrm{al}^{37}$ have also reported increased matrix metalloproteinase MMP-9 activity downstream of UPA/UPAR signaling in diabetic mice retina which leads to ECM disruption in retinal vessels. Absence of $\mathrm{UPAR}^{\text {in }} \mathrm{uPAR}^{-1-}$ mice results in blockade of diabetes-induced increase in retinal vascular permeability and MMP-9 activity. Interestingly, VEGF can also directly influence levels of UPAR receptor and increase the permeability of retinal endothelial cells through the uPAR-GSK3 $\beta / \beta$ catenin signaling pathway. ${ }^{37}$ Taken together, these reports suggest that retinal inflammation induces endothelial dysfunction and apoptosis, which leads to microthrombosis and hemorrhage. In addition, the extravasation of activated coagulation factors can further promote retinal inflammation and endothelial dysfunction (-Fig. 2).

Multiple studies have reported that anti-inflammatory interventions, including salicylates, decreased nuclear translocation of NF-KB, expression of proinflammatory proteins, apoptosis, and capillary loss in diabetic rat retina. ${ }^{8}$ The majority of clinical studies have not detected a significant effect of aspirin on diabetic retinopathy, reviewed by

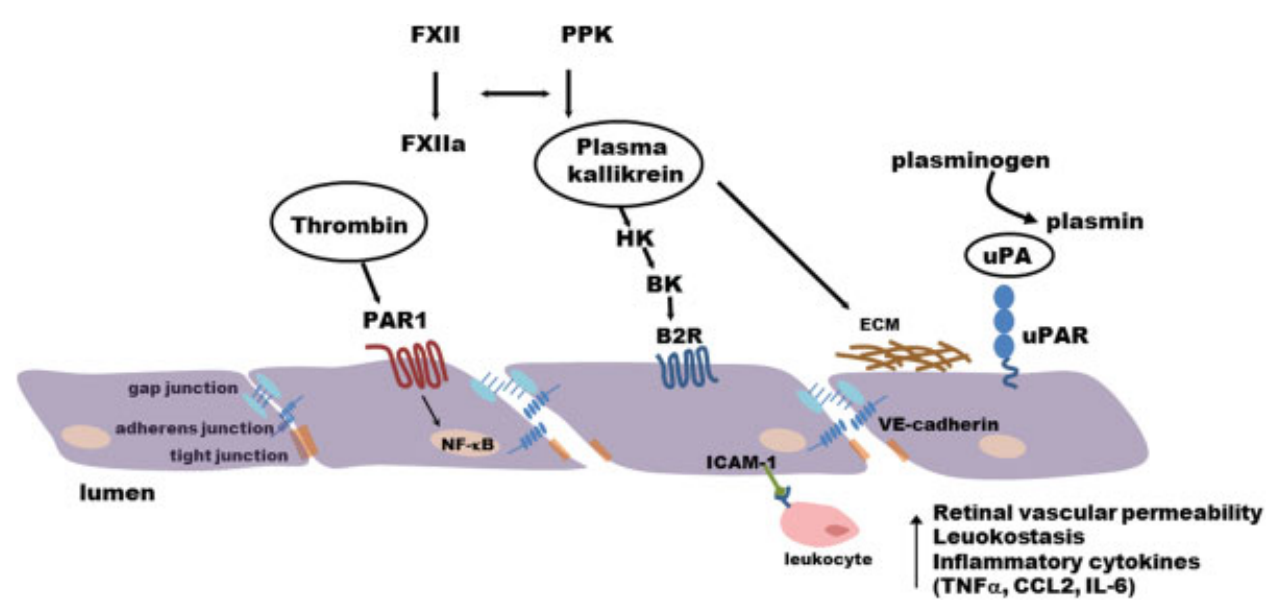

Fig. 2 Activated coagulation factors promote retinal vascular permeability, leukostasis, and inflammatory cytokines. Extravasation increases intraocular levels of thrombin, plasma kallikrein, and uPA, which trigger inflammatory processes in the retina. Thrombin signals via proteaseactivated receptor 1 (PAR1) and NF-KB to induce inflammatory cytokines. Plasma kallikrein generates bradykinin, which can signal through B2R to increase vascular permeability, leukostasis, and edema. Plasma kallikrein also has collagenase-like activity that mediates extracellular matrix (ECM) degradation. Active UPA/UPAR signaling mediates disruption of the blood retinal barrier through proteolytic cleavage of VE-cadherin. 
Bergerhoff et al. ${ }^{39}$ However, recent findings from the MADIABETES study reported an association of aspirin use in patients with type 2 diabetes with an increased incidence of diabetic retinopathy. ${ }^{40}$ While salicylates and other antiinflammatory molecules have been shown to exert protective effects on the retina in diabetic animal models, the specific clinical effects of this therapeutic approach on retinal inflammation, thrombosis, and hemostasis are not yet available.

\section{Diabetes and Retinal Vein Occlusion}

Retinal vein occlusion is an important cause of retinal vascular disease and vision loss, with an overall incidence in of approximately 1 to $2 \%$ in people older than 40 years. ${ }^{41}$ Santiago et $\mathrm{al}^{42}$ have examined the potential effects of diabetes on the prevalence of central retinal vein occlusion (CRVO) and its outcomes. This retrospective chart review of 19,648 subjects over a 4-year period showed that CRVO was observed at a similar prevalence in diabetic and nondiabetic subjects; however, diabetes was associated with worse anatomical outcomes, including disc neovascularization and panretinal photocoagulation. CRVO patients with diabetes in this study included both type 1 and type 2 diabetics with $27.1 \pm 10$ and $12.1 \pm 6.7$ years of diabetes duration, respectively. Thus, the effects of diabetes on CRVO outcome may be mediated, in part, by retinal vascular abnormalities associated with diabetic retinopathy. While the prevalence of CRVO is increased in several hypercoagulable conditions, reviewed in a study by MacDonald, ${ }^{41}$ diabetes appears to worsen CRVO outcomes rather than increase its incidence.

\section{Conclusion}

Capillary nonperfusion and intraretinal hemorrhage are associated with advanced stages of diabetic retinopathy. Although increased prevalence of microthrombi has been detected in the diabetic retina, their significance to retinal ischemia is unknown. In contrast, marked increases in coagulation and fibrinolytic factors, including plasma kallikrein, thrombin, and urokinase, have been detected in ocular samples from patients with advanced diabetic retinopathy. In addition to their roles in thrombosis and hemostasis, these factors promote retinal inflammation, vascular dysfunction, and proteolytic disruption of ECM and intercellular junctional complexes. Intraocular hemorrhage, endothelial dysfunction, and breakdown of the blood retinal barrier appear to contribute to the extravasation and activation of coagulation components in the retina and vitreous in diabetes. Inhibition of these thromboinflammatory pathways may provide therapeutic opportunities for diabetic retinopathy, especially for advanced stages of this disease.

\section{Acknowledgments}

This article was supported by the National Institutes of Health (NIH) Award Numbers EY019029, DK36836, and T32EY007145. The content is solely the responsibility of the authors and does not necessarily represent the official views of the NIH.

\section{References}

1 Yau JW, Rogers SL, Kawasaki R, et al; Meta-Analysis for Eye Disease (META-EYE) Study Group. Global prevalence and major risk factors of diabetic retinopathy. Diabetes Care 2012;35(3):556-564

2 Antonetti DA, Klein R, Gardner TW. Diabetic retinopathy. N Engl J Med 2012;366(13):1227-1239

3 Aiello LP, Avery RL, Arrigg PG, et al. Vascular endothelial growth factor in ocular fluid of patients with diabetic retinopathy and other retinal disorders. N Engl J Med 1994;331(22):1480-1487

4 Grant PJ. Diabetes mellitus as a prothrombotic condition. J Intern Med 2007;262(2):157-172

5 Grant PJ, Kruithof EKO, Felley CP, Felber JP, Bachmann F. Short-term infusions of insulin, triacylglycerol and glucose do not cause acute increases in plasminogen activator inhibitor-1 concentrations in man. Clin Sci (Lond) 1990;79(5):513-516

6 Liu J, Feener EP. Plasma kallikrein-kinin system and diabetic retinopathy. Biol Chem 2013;394(3):319-328

7 Hammes HP, Lin J, Renner O, et al. Pericytes and the pathogenesis of diabetic retinopathy. Diabetes 2002;51(10):3107-3112

8 Zheng L, Howell SJ, Hatala DA, Huang K, Kern TS. Salicylate-based anti-inflammatory drugs inhibit the early lesion of diabetic retinopathy. Diabetes 2007;56(2):337-345

9 Hammes HP, Lin J, Wagner P, et al. Angiopoietin-2 causes pericyte dropout in the normal retina: evidence for involvement in diabetic retinopathy. Diabetes 2004;53(4):1104-1110

10 Miyamoto K, Khosrof S, Bursell SE, et al. Prevention of leukostasis and vascular leakage in streptozotocin-induced diabetic retinopathy via intercellular adhesion molecule- 1 inhibition. Proc Natl Acad Sci U S A 1999;96(19):10836-10841

11 Iliaki E, Poulaki V, Mitsiades N, Mitsiades CS, Miller JW, Gragoudas ES. Role of alpha 4 integrin (CD49d) in the pathogenesis of diabetic retinopathy. Invest Ophthalmol Vis Sci 2009;50(10):4898-4904

12 Phipps JA, Clermont AC, Sinha S, Chilcote TJ, Bursell SE, Feener EP. Plasma kallikrein mediates angiotensin II type 1 receptor-stimulated retinal vascular permeability. Hypertension 2009;53(2): 175-181

13 Abiko T, Abiko A, Clermont AC, et al. Characterization of retinal leukostasis and hemodynamics in insulin resistance and diabetes: role of oxidants and protein kinase-C activation. Diabetes 2003; 52(3):829-837

14 Noda K, Nakao S, Zandi S, Sun D, Hayes KC, Hafezi-Moghadam A. Retinopathy in a novel model of metabolic syndrome and type 2 diabetes: new insight on the inflammatory paradigm. FASEB J 2014;28(5):2038-2046

15 Miyamoto K, Khosrof S, Bursell SE, et al. Vascular endothelial growth factor (VEGF)-induced retinal vascular permeability is mediated by intercellular adhesion molecule-1 (ICAM-1). Am J Pathol 2000;156(5):1733-1739

16 Ramírez M, Wu Z, Moreno-Carranza B, et al. Vasoinhibin gene transfer by adenoassociated virus type 2 protects against VEGFand diabetes-induced retinal vasopermeability. Invest Ophthalmol Vis Sci 2011;52(12):8944-8950

17 Tolentino MJ, Miller JW, Gragoudas ES, et al. Intravitreous injections of vascular endothelial growth factor produce retinal ischemia and microangiopathy in an adult primate. Ophthalmology 1996;103(11):1820-1828

18 Campochiaro PA, Wykoff CC, Shapiro H, Rubio RG, Ehrlich JS. Neutralization of vascular endothelial growth factor slows progression of retinal nonperfusion in patients with diabetic macular edema. Ophthalmology 2014;121(9):1783-1789

19 Yamashiro K, Tsujikawa A, Ishida S, et al. Platelets accumulate in the diabetic retinal vasculature following endothelial death and 
suppress blood-retinal barrier breakdown. Am J Pathol 2003; 163(1):253-259

20 Boeri D, Maiello M, Lorenzi M. Increased prevalence of microthromboses in retinal capillaries of diabetic individuals. Diabetes 2001;50(6):1432-1439

21 Vinik AI, Erbas T, Park TS, Nolan R, Pittenger GL. Platelet dysfunction in type 2 diabetes. Diabetes Care 2001;24(8):1476-1485

22 Matsubara Y, Murata M, Maruyama T, et al. Association between diabetic retinopathy and genetic variations in alpha2beta1 integrin, a platelet receptor for collagen. Blood 2000;95(5):1560-1564

23 Gao BB, Clermont A, Rook S, et al. Extracellular carbonic anhydrase mediates hemorrhagic retinal and cerebral vascular permeability through prekallikrein activation. Nat Med 2007;13(2):181-188

24 Gao BB, Chen X, Timothy N, Aiello LP, Feener EP. Characterization of the vitreous proteome in diabetes without diabetic retinopathy and diabetes with proliferative diabetic retinopathy. J Proteome Res 2008;7(6):2516-2525

25 Clermont A, Chilcote TJ, Kita T, et al. Plasma kallikrein mediates retinal vascular dysfunction and induces retinal thickening in diabetic rats. Diabetes 2011;60(5):1590-1598

26 Liu J, Clermont AC, Gao BB, Feener EP. Intraocular hemorrhage causes retinal vascular dysfunction via plasma kallikrein. Invest Ophthalmol Vis Sci 2013;54(2):1086-1094

27 Liu J, Gao BB, Feener EP. Proteomic identification of novel plasma kallikrein substrates in the astrocyte secretome. Transl Stroke Res 2010;1(4):276-286

28 Liu J, Gao BB, Clermont AC, et al. Hyperglycemia-induced cerebral hematoma expansion is mediated by plasma kallikrein. Nat Med 2011;17(2):206-210

29 Abdallah RT, Keum JS, El-Shewy HM, et al. Plasma kallikrein promotes epidermal growth factor receptor transactivation and signaling in vascular smooth muscle through direct activation of protease-activated receptors. J Biol Chem 2010;285(45): 35206-35215

30 Chahed S, Leroyer AS, Benzerroug M, et al. Increased vitreous shedding of microparticles in proliferative diabetic retinopathy stimulates endothelial proliferation. Diabetes 2010;59(3):694-701

31 Van Der Meijden PE, Van Schilfgaarde M, Van Oerle R, Renné T, ten Cate H, Spronk HM. Platelet- and erythrocyte-derived micropar- ticles trigger thrombin generation via factor XIIa. J Thromb Haemost 2012;10(7):1355-1362

32 Müller F, Mutch NJ, Schenk WA, et al. Platelet polyphosphates are proinflammatory and procoagulant mediators in vivo. Cell 2009; 139(6):1143-1156

33 Bastiaans J, van Meurs JC, Mulder VC, et al. The role of thrombin in proliferative vitreoretinopathy. Invest Ophthalmol Vis Sci 2014; 55(7):4659-4666

34 Bastiaans J, van Meurs JC, van Holten-Neelen C, et al. Factor Xa and thrombin stimulate proinflammatory and profibrotic mediator production by retinal pigment epithelial cells: a role in vitreoretinal disorders? Graefes Arch Clin Exp Ophthalmol 2013;251(7): 1723-1733

35 Cowan C, Muraleedharan CK, O'Donnell JJ III, et al. MicroRNA-146 inhibits thrombin-induced NF- $\mathrm{KB}$ activation and subsequent inflammatory responses in human retinal endothelial cells. Invest Ophthalmol Vis Sci 2014;55(8):4944-4951

36 Das A, McGuire PG, Eriqat C, et al. Human diabetic neovascular membranes contain high levels of urokinase and metalloproteinase enzymes. Invest Ophthalmol Vis Sci 1999;40(3):809-813

37 El-Remessy AB, Franklin T, Ghaley N, et al. Diabetes-induced superoxide anion and breakdown of the blood-retinal barrier: role of the VEGF/uPAR pathway. PLoS ONE 2013;8(8):e71868

38 Navaratna D, Menicucci G, Maestas J, Srinivasan R, McGuire P, Das A. A peptide inhibitor of the urokinase/urokinase receptor system inhibits alteration of the blood-retinal barrier in diabetes. FASEB J 2008;22(9):3310-3317

39 Bergerhoff K, Clar C, Richter B. Aspirin in diabetic retinopathy. A systematic review. Endocrinol Metab Clin North Am 2002;31(3): 779-793

40 Salinero-Fort MA, San Andrés-Rebollo FJ, de Burgos-Lunar C, Arrieta-Blanco FJ, Gómez-Campelo P; MADIABETES GroupFouryear incidence of diabetic retinopathy in a Spanish cohort: the MADIABETES study. PLoS ONE 2013;8(10):e76417

41 MacDonald D. The ABCs of RVO: a review of retinal venous occlusion. Clin Exp Optom 2014;97(4):311-323

42 Santiago JG, Walia S, Sun JK, et al. Influence of diabetes and diabetes type on anatomic and visual outcomes following central rein vein occlusion. Eye (Lond) 2014;28(3):259-268 